designing new structures would be a hit-or-miss affair.

Interest in the solution of the problem of analysis and, subsequently, prediction of protein structure from sequence has an extensive history. From the studies of Ramachandran (J. molec. Biol. 7, 95; 1963), to those of Levitt (Nature 261, 552; 1976; J. molec. Biol. 104, 59; 1976) theoretically oriented work has been progressing. The basic data for these studies have been the protein structures which regularly appear in the literature. The problem has tended to be approached from the point of view of overall structure with atomic details being left until later.

This has been partly due to the fact that most protein structures available are unrelated. Of the related structures the globins are probably the ones likely to reveal the greatest comparative information. The overall picture of haemoglobin and its allostery has been described by Perutz. Not only is there an abundance of species and withinspecies mutant haemoglobins of the four-chain type, but also single chain oxygen carriers such as haemerythrin (Proc. natn. Acad. Sci. U.S.A. 72, 2160; 1975), erythrocruorin (Eur. J. Biochem. $19,42 ; 1971)$ and the monomer/dimer system of the sea lamprey ( $J$. molec. Biol. 74, 331; 1973). Consideration of the chain interactions in atomic detail is likely to be stimulated by recent refinement of sperm whale myoglobin.

Takano (J. molec. Biol. 110, 533, $569 ; 1977)$ has just published the first refinement of the deoxy- and metstructures since Kendrew et al. (Nature 190, 666; 1961) first interpreted their map. Using new diffractometer data and variants of the well-tried method of alternating phase and realspace refinement (Acta Cryst. A27, 436; 1971) structures suitable for detailed comparison with haemoglobin were achieved. Takano's stereo diagrams show rather clearly the similarity of structure despite the differences in sequence. The possibility of extensive comparisons of this type is most encouraging, but it does raise a number of problems. Should one compare only whole myoglobin molecules or also separated sections of the chain? How can one objectively make the comparisons between non-identical objects and quantify and display the comparisons?

The relationship between haemoglobin and myoglobin provides information at all levels, but the relative changes are greater than one might want for truly secondary structure. As a starting point movements smaller than those produced by a single amino acid change would be interesting. Such movements would be expected to occur immediately before denaturation, even though it is a cooperative phenomenon. Stability studies on haemoglobin have already been started (see Nature 247, 341; 1974; Nature 255, 256; 1975). Extension to full crystallographic refinements with crystals at, for example, different $p \mathrm{H}$ and ionic strengths might be the key to a number of problems associated with small atomic movements. But will there be PhD students to do all those structures?

\section{Heat flow at the mantle surface}

\section{from Peter J. Smith}

Some time ago Chapman and Pollack (Earth planet. Sci. Lett. 28, 23; 1975) took what they called "a new look" at the Earth's thermal characteristics, producing in the process the first worthwhile map of the global surface heat flow. Then, as now, there were still large areas of the world with few or no data; but Chapman and Pollack had hit upon the idea of filling these gaps with heat flow values predicted from known empirical relationships between heat flow and tectonic age. Both real and predicted observations were then incorporated into a 12th degree spherical harmonic analysis which was translated into a global contour map.

Pollack and Chapman (Earth planet. Sci. Lett. 34, 174; 1977) have now extended their analysis to the much more difficult problem of plotting the heat flow at the crust-mantle boundary. Mantle heat flow is, of course, the total heat flow less that produced or released in the crust; but it is not easy to determine just what the crustal contribution is. Most heat produced in the continental crust comes from the decay of radioactive isotopes whose concentration decreases with depth in some only vaguely understood way. Most heat from the oceanic crust, on the other hand, is released as the lithosphere produced at oceanic ridges cools, although there is also a small but significant radiogenic component.

Combining theory with experimental results, Pollock and Chapman nevertheless manage to estimate these crustal contributions to the total heat flow, whence they derive sufficient data to carry out an 18 th degree spherical harmonic analysis. The resulting global contour map of heat flow at the mantle

Peter J. Srnith is in the Department of Earth Sciences at the Open University. surface differs from the corresponding crustal surface map in two notable respects. First, a comparison makes it clear that the near equality of continental and oceanic heat flows at the Earth's surface does not extend down to the mantle; at the crust-mantle boundary the mean heat flow in continental regions is only about half of that in oceanic regions $\left(28 \mathrm{~mW} \mathrm{~m}^{-2}\right.$ and $57 \mathrm{~mW} \mathrm{~m}^{-2}$, respectively). This means that the mantle below the oceans must be considerably hotter than that beneath the continents as several theoretical models have already suggested (see for example, MacDonald Rev. Geophys. Space Phys. 1, 587; 1963).

The second result is that there is much less heat flow contrast within continental regions at the mantle surface than there is at the crustal surface. In Australia, for example, the mean crustal surface heat flow is $81 \mathrm{~mW} \mathrm{~m}^{-2}$ in the Proterozoic rocks of the central region and $39 \mathrm{~mW} \mathrm{~m}^{-2}$ in the western shield. At the mantle level both regions have heat flows of $27 \mathrm{~mW} \mathrm{~m}^{-2}$. Evidently within-continent heat flow variations at the Earth's surface are due entirely, or almost entirely, to regional differences in crustal radioactivity and/or erosion. At the crust-mantle boundary the heat flow contours are simple closed curves centred on the continents and showing no regional complexities.

Finally, Pollack and Chapman are able to demonstrate correlations between the heat flow fields and other geophysical parameters. For example, both total and mantle heat flows correlate positively with the Earth's gravitational potential. However, the mantle heat flow correlates the less positively, suggesting that the broad geographic variations in the gravity field may be due in part to thermally produced heterogeneities in the crust. On the other hand, the fact that the positive correlation remains when the crustal contribuiton is removed suggests that thermal heterogeneities extend well into the mantle.

By contrast, both the total and mantle heat flows correlate negatively (the latter the more so) with topography, although this is produced by the first-order topography of the Earth (the gross difference in levels between continental and oceanic crust) which is largely a petrological rather than a thermal effect. When the continental and oceanic levels are normalised to remove this petrologically produced contrast, the total and mantle heat flows are found to correlate positively with topography, emphasising, according to Pollack and Chapman, "the significant role that temperature plays in the morphology of both continents and oceans." 